Pacific Journal of Mathematics

MINIMAL URYSOHN SPACES 


\section{MINIMAL URYSOHN SPACES}

\section{T. Scarborough}

A topological space $X$ is (1) $H$ (i), (2) $H$ (ii), (3) $R$ (i), (4) $R$ (ii) if (1) Every open filter on $X$ has nonvoid adherence, (2) Every open filter on $X$ with one-point adherence is convergent, (3) Every regular filter on $X$ has nonvoid adherence, (4) Every regular filter on $X$ with one point adherence is convergent. These properties, which were investigated by Scarborough and Stone in a recent paper, arose naturally from the study of minimal Hausdorff, $H$-closed, minimal regular and $R$-closed spaces. This paper investigates similar properties for minimal Urysohn and Urysohn closed spaces.

Urysohn filters are introduced and another characterization of minimal Urysohn and Urysohn closed spaces is obtained. In connection with product spaces, it is shown that in some sense open filters and regular filters have more in common with each other than either has with Urysohn filters, dispite the fact that Urysohn filters "lie between" these first two types.

A topological space $X$ is said to be Urysohn if every two distinct points of $X$ can be separated by disjoint closed neighborhoods. A space $(X, \mathscr{T})$ is said to be minimal Urysohn if $\mathscr{T}$ is a Urysohn topology on $X$ and there is no strictly smaller Urysohn topology contained in $\mathscr{T}$. A space $(X, \mathscr{T})$ is said to be Urysohn closed ( $U$-closed) if $\mathscr{T}$ is a Urysohn topology and $X$ is closed in every Urysohn space in which it is embedded. Herrlich [5] has shown the existence of noncompact minimal Urysohn spaces and also of $U$-closed spaces which are not minimal Urysohn. ${ }^{1}$ In what follows, we generalize the notions of minimal Urysohn and $U$-closed and extend them to spaces which are not necessarily Urysohn (or even Hausdorff).

A filter base $\mathscr{F}$ on a space $X$ is said to be an open filter if each member of $\mathscr{F}$ is an open set. An open filter $\mathscr{F}$ is said to be a Urysohn filter or a $U$-filter if given any point $x$ not in the adherence of $\mathscr{F}$, there exists a neighborhood $U$ of $x$ and an $F \in \mathscr{F}$ such that $U^{\prime} \cap F^{\prime}=\varnothing\left(A^{\prime}\right.$ denotes the closure of the set $\left.A\right)$. Using the same techniques as in [1] or [3], we see that the following is true:

(1) A Urysohn space is $U$-closed if and only if every $U$-filter has nonvoid adherence.

(2) A Urysohn space is minimal Urysohn if and only if every $U$-filter with one-point adherence converges to this point.

We say that a space $X$ is a $U(i)$ space or is $U(i)$ if every $U$-filter

\footnotetext{
1 The existence and characterizations of these spaces were also known to the author.
} 
on $X$ has nonvoid adherence. We also say that a space $X$ is a $U$ (ii) space or is $U$ (ii) if every $U$-filter on $X$ with one-point adherence converges to this point. A space can be $U$ (ii) is two ways; it can satisfy the condition $U$ (ii) vacuously or nonvacuously. Thus the statement " $X$ is $U$ (ii) vacuously" will mean that no $U$-filter on $X$ has a unique adherent point, and the statement " $X$ is $U$ (ii) nonvacuously" will mean that $X$ is $U$ (ii) and also there is at least one $U$-filter on $X$ with onepoint adherence. This last requirement is certainly met if $X$ is a nonempty Urysohn space. Every minimal Urysohn space is $U$-closed, and by an argument analagous to that in [3, p. 98], it follows that a space which is $U($ ii) nonvacuously is also $U(\mathrm{i})$.

For an example of a countable $T_{1}$ space which satisfies $U(i i)$ vacuously but does not satisfy $U(\mathrm{i})$ see [7, Ex 2.2], and for an example of a countable $T_{1}$ space which satisfies $U$ (ii) nonvacuously, see [7, Ex 2.3].

If $\left\{X_{a}: a \in A\right\}$ is a family of spaces, we write $\pi X_{a}$ for the Cartesian product of this family, and assume $\pi X_{a}$ is equipped with the product topology. We also assume that no factor is empty. The notation and definitions will be that of [7].

THEOREM 1. If $X=\pi X_{a}$ is $U(\mathrm{i})$, then each factor is $U(\mathrm{i})$.

This follows from the observation that the continuous image of a $U(\mathrm{i})$ space is $U(\mathrm{i})$.

In particular, if $\pi X_{a}$ is $U$-closed, then so is each $X_{a}$. It is unknown whether the converse of the above theorem is true or not, even when all the factors are $U$-closed. However, in certain special cases we are able to give an affirmative answer.

Lemma 2. Let $X$ be an $H(\mathrm{i})$ space and $Y$ an arbitrary space. If $\mathscr{F}$ is a U-filter on $X \times Y$, then $\pi_{2} \mathscr{F}$ is a U-filter on $Y$.

If $y \notin a d \pi_{2} \mathscr{F}$, then for each $x \in X$, there exist neighborhoods $U_{x}$ and $V_{x}$ of $x$ and $y$ respectively and an $F_{x} \in \mathscr{F}$ such that

$$
\left(U_{x}^{\prime} \times V_{x}^{\prime}\right) \cap F_{x}^{\prime}=\varnothing .
$$

Since $X$ is $H(\mathrm{i})$, there exist $x_{1}, \cdots, x_{n} \in X$ such that

$$
\cup\left\{U_{x_{k}}^{\prime}: 1 \leqq k \leqq n\right\} \supset X ;
$$

see [6, p. 132]. Thus

$$
\left(X \times \cap\left\{V_{x_{k}}^{\prime}: 1 \leqq k \leqq n\right\}\right) \cap\left(\cap\left\{F_{x_{k}}^{\prime}: 1 \leqq k \leqq n\right\}\right)=\varnothing,
$$

and it follows from $[7,2.17]$ that

$$
\left(\cap\left\{V_{x_{k}}^{\prime}: 1 \leqq k \leqq n\right\}\right) \cap\left(\pi_{2} \cap\left\{F_{x_{k}}: 1 \leqq k \leqq n\right\}\right)^{\prime}=\varnothing .
$$


We have produced a closed neighborhood $\cap\left\{V_{x_{k}}^{\prime}: 1 \leqq k \leqq n\right\}$ of $y$ which fails to meet the closure of $\pi_{2} \cap\left\{F_{x_{k}}: 1 \leqq k \leqq n\right\}$; whence $\pi_{2} \mathscr{F}$ is a $U$-filter.

LEMma 3. Let $X$ be an $H(\mathrm{i})$ space and $Y$ be an arbitrary space. If $\mathscr{F}$ is an open filter on $X \times Y$ and if $y \in a d \pi_{2} \mathscr{F}$, then there exists an $x \in X$ such that $(x, y) \in a d \mathscr{F}$.

The proof is essentially that of $[7,3.13]$ and is omitted.

THEOREM 4. If $X$ is $H(\mathrm{i})$ and $Y$ is $U(\mathrm{i})$, then $X \times Y$ is $U(\mathrm{i})$.

The proof follows immediately from Lemma 2 and 3 .

CoROllary 5. The product of a compact Hausdorff space (or equivalently, a regular $U$-closed space) with a U-closed space is $U$ closed.

We now consider the question as to whether theorems similar to 2.9 and 3.8 of [7] also hold for $U$ (ii) spaces. In view of these theorems and the fact that every regular filter is a $U$-filter and every $U$-filter is an open filter, it might be conjectured that a corresponding theorem is also valid for $U$ (ii) spaces. The following theorem and example completely resolve this question.

Theorem 6. If $X=\pi X_{a}$, and $X$ satisfies $U(\mathrm{ii})$ vacuously, then at least one $X_{a}$ does so.

If no $X_{a}$ satisfies $U\left(\right.$ ii) vacuously, then there exists a $U$-filter $\mathscr{F}_{a}$ on each $X_{a}$ with a unique adherent point $x_{a}$. The product filter $\pi \mathscr{F}_{a}$ is a $U$-filter on $X$ with the unique adherent point $\pi x_{a}$. This is a contradiction.

Next we present an example of a product space $X_{1} \times X_{2}$ in which $X_{2}$ is $U$ (ii) vacuously while $X_{1} \times X_{2}$ is not $U$ (ii) vacuously (it is not $U(\mathrm{ii}))$.

Example 7. Let $I$ be the positive integers. Let $X_{1}$ be the Urysohn subspace of $X$ (described in [3, p. 98]) consisting of the points $a_{i j}, a$ where $i, j \in I$.

We next describe $X_{2}$. Let $S$ be the set of all functions from $I$ into $I$. Let

$$
\begin{aligned}
X_{2} & =\left\{a_{i j}^{\prime}, b_{i j}^{\prime}, c_{i j}^{\prime}, d_{i j}^{\prime}, e_{i}^{\prime}, f_{i}^{\prime}: i, j \in I\right\} \cup\left\{a^{\prime}, b^{\prime}\right\}, \\
A_{i n} & =\left\{a_{i j}^{\prime}, b_{i j}^{\prime}: j \geqq n\right\} \text { and } B_{i n}=\left\{c_{i j}^{\prime}, d_{i j}^{\prime}: j \geqq n\right\}
\end{aligned}
$$


where all the symbols $a_{i j}^{\prime}, b_{i j}^{\prime}, c_{i j}^{\prime}, d_{i j}^{\prime}, e_{i}^{\prime}, f_{i}^{\prime}, a^{\prime}, b^{\prime}$ are assumed to represent distinct points. If we let

$$
\begin{gathered}
\mathscr{B}=\left\{\left\{a_{i j}^{\prime}, b_{i j}^{\prime}\right\},\left\{c_{i j}^{\prime}, d_{i j}^{\prime}\right\}: i, j \in I\right\} \cup\left\{\left\{e_{i}^{\prime}, f_{i}^{\prime}\right\} \cup A_{i n} \cup B_{i m}: i, n, m \in I\right\} \\
\cup\left\{\left\{a^{\prime}\right\} \cup \cup\left\{A_{i f(i)}: i \geqq n\right\}: n \in I, f \in S\right\} \\
\cup\left\{\left\{b^{\prime}\right\} \cup \cup\left\{B_{i f(i)}: i \geqq n\right\}: n \in I, f \in S\right\},
\end{gathered}
$$

then $\mathscr{B}$ is a base for a topology on $X_{2}$ which is $U$ (ii) vacuously.

We will show that $X_{1} \times X_{2}$ is not $U$ (ii) by exhibiting a $U$-filter on $X_{1} \times X_{2}$ with a unique adherent point to which it does not converge.

For each $n \in I$, let

$$
F_{n}=\bigcup\left\{\left\{a_{i j}\right\} \times\left\{a_{i j}^{\prime}, b_{i j}^{\prime}\right\}: j \in I, i \geqq n\right\} .
$$

Clearly $\mathscr{F}=\left\{F_{n}: n \in I\right\}$ is an open filter on $X_{1} \times X_{2}$ with $\left(a, a^{\prime}\right)$ as its only point of adherence. Since $F_{n}^{\prime}=F_{n} \cup\left\{\left(a, a^{\prime}\right)\right\}, \mathscr{F}$ is a $U$-filter on $X_{1} \times X_{2}$ with a unique adherent point to which it does not converge. Thus $X_{1} \times X_{2}$ is not $U$ (ii), but is $R$ (ii) vacuously by Theorem 3.8 of [7] in view of the fact that every regular filter base is a $U$-filter.

Because of the above example, it is not possible to use the same technique as was employed in Theorem 3.9 of [7] to prove the following conjecture: If $X=\pi X_{a}$ is $U$ (ii) nonvacuously, then each $X_{a}$ is $U$ (ii) nonvacuously. Using the same idea as in Theorem 6 , it is easy to see that some $X_{a}$ must be $U(\mathrm{ii})$, but the above conjecture as well as its converse remain unsolved problems. Indeed, it is not known whether or not the product of minimal Urysohn spaces is minimal Urysohn. However, in certain special cases we were able to obtain the following results.

Theorem 8. If $X \times Y$ is $U(\mathrm{ii})$ and if $Y$ has a Urysohn filter $\mathscr{G}$ with a unique adherent point $y$, then $X$ is $U(\mathrm{ii})$.

Let $\mathscr{F}$ be a $U$-filter on $X$ with a unique adherent point $x$. Then $\mathscr{F} \times \mathscr{G}$ is a $U$-filter on $X \times Y$ with a unique adherent point $(x, y)$. Since $X \times Y$ is $U$ (ii), $\mathscr{F} \times \mathscr{G}$ converges to $(x, y)$; whence $\mathscr{F}$ converges to $x$. Thus $X$ is $U$ (ii).

CoROllary 9. If $X=\pi X_{a}$ is minimal Urysohn, then each factor is minimal Urysohn.

Let $X_{b}$ be a factor and write $Y$ for the product of the rest. Then $Y$ is a nonempty Urysohn space (whenever we write $\pi X_{a}$, we assume that each $\left.X_{a} \neq \varnothing\right)$. Let $y \in Y$ and $\mathscr{N}(y)$ be the open neighborhood system of $y$. By the preceding theorem, $X_{b}$ is $U$ (ii); thus $X_{b}$ is minimal Urysohn. 
THEOREM 10. If $X$ is $H(\mathrm{ii})$ nonvacuously and $Y$ is $U(\mathrm{ii})$, then $X \times Y$ is $U($ ii).

Let $\mathscr{F}$ be a $U$-filter on $X \times Y$ with a unique adherent point $(x, y)$. By Lemma $2, \pi_{2} \mathscr{F}$ is a $U$-filter on $Y$ and by Lemma $3, y$ is the only adherent point of $\pi_{2} \mathscr{F}$. Thus $\pi_{2} \mathscr{F}$ converges to $y$. If $w \in a d \pi_{1} \mathscr{F}$, then $(w, y) \in a d \mathscr{F}$, so $w=x$. Hence $\pi_{1} \mathscr{F}$ is an open filter on an $H$ (ii) space with a unique adherent point $x$ to which it must converge. Thus $\mathscr{F}$ converges to $(x, y)$, so $X \times Y$ is $U$ (ii).

CoROLlary 11. If $X$ is compact Hausdorff and $Y$ is minimal Urysohn, then $X \times Y$ is minimal Urysohn.

The proof is immediate from the preceding theorem.

Next we show that there are large numbers of $U$-closed spaces which are not minimal Urysohn and also large numbers of minimal Urysohn spaces which are not compact. Corresponding theorems for the Hausdorff and regular cases are found in [7].

THEOREM 12. There exist U-closed spaces of every infinite cardinality which are not minimal Urysohn.

Let $X_{1}$ be the Urysohn subspace of $X$ (described in [3, p. 98]) consisting of the points $a_{i j}, c_{i}, a, i, j \in I . \quad X_{1}$ is a countable absolutely closed Urysohn space which is not minimal Urysohn. Thus $X_{1}$ is $U$ closed. Given an infinite cardinal $K$, let $Z$ be a compact Hausdorff space of cardinality $K$-for instance let $Z$ be the one point compactification of a discrete space of cardinal $K$. By Corollary $5, X_{1} \times Z$ is $U$-closed and obviously has cardinal $K$. By Corollary $9, X_{1} \times Z$ is not minimal Urysohn, although this is easy to verify directly.

THEOREM 13. There exist minimal Urysohn spaces of every uncountably infinite cardinality which are not compact.

By Corollary 11 and the proof of Theorem 12 it suffices to exhibit a noncompact minimal Urysohn space with cardinality equal to the first uncountable cardinal. Such a space is found in [5, p. 289].

Every countable $R$-closed space is compact [7, p. 137], so every countable minimal regular space is compact. There exist countable absolutely closed spaces which are not minimal Hausdorff (see the proof of 12) and also noncompact minimal Hausdorff spaces which are countable; see [3, p. 98]. Do there exist noncompact minimal Urysohn spaces which are countable? 
Berri [4] has shown that every minimal Hausdorff space which is countable has an isolated point. The same reasoning also shows that every countable absolutely closed space has an isolated point. With trivial modifications of this proof the following theorem results.

THEOREM 14. Every countable U-closed space has an isolated point.

CoRollary 15. Every countable minimal Urysohn space has an isolated point.

It is well known [6] that absolutely closed plus semiregular is equivalent to minimal Hausdorff. The relation of semiregularity to the corresponding properties in the Urysohn case is given in the following.

TheOREM 16. A minimal Urysohn space $X$ is semiregular.

If $X$ is not semiregular at some point $x \in X$, then

$$
\mathscr{F}=\left\{\left(N^{\prime}\right)^{0}: N \in \mathscr{N}(x)\right\}
$$

is a $U$-filter on $X$ having a unique adherent point to which it does not converge. This is a contradiction.

CoRollary 17. Every minimal Urysohn space can be embedded densely in a minimal Hausdorff space.

The result follows immediately from the fact that a space is semiregular if and only if it can be densely embedded in a minimal Hausdorff space; see [2].

It is not true that semiregularity plus $U$-closure implies minimal Urysohn as the following example shows.

EXAmPLE 18. Let $X$ be the subspace consisting of $\{a\} \cup R_{1} \cup R_{2}$ in the notation of [5, Ex 4]. Then $X$ is $U$-closed and semiregular but not minimal Urysohn.

\section{REFERENCES}

1. B. Banaschewski, Über zwei Extremaleigenschaften topologischer Räume, Math, Nachr. 13 (1955), 141-150.

2. — Über Hausdorffsch-minimale Erweiterung von Räume, Arch. Math. 12 (1961), 355-365.

3. M. P. Berri, Minimal topological spaces, Trans. Amer. Math. Soc. 108 (1963), 97105.

4. - Categories of certain minimal topological spaces, J. Austral Math. Soc. 
4 (1964), 78-82.

5. H. Herrlich, Tע-Abgeschlossenheit und Tע-Minimalität, Math. Z. 88 (1965), 285-294

6. M. Katětov., Über H-abgeschlossene und bikompakte Räume, Časopis Pěst. Mat. 69 (1940), 36-49.

7. C. T. Scarborough and A. H. Stone, Products of nearly compact spaces, Trans. Amer. Math. Soc. 124 (1966), 131-147.

Received June 30, 1967. This research was partially supported by a grant from the State of Mississippi.

Mississippi State UNIVERSity

State College, Mississippi 



\section{PACIFIC JOURNAL OF MATHEMATICS}

\section{EDITORS}

\section{H. ROYDEN}

Stanford University

Stanford, California

\section{J. Dugundu}

Department of Mathematics University of Southern California Los Angeles, California 90007

RICHARD ARENS

University of California Los Angeles, California 90024

ASSOCIATE EDITORS
E. F. Beckenbach
B. H. NEUMANN
F. WoLF
K. YosidA

\section{SUPPORTING INSTITUTIONS}

\author{
UNIVERSITY OF BRITISH COLUMBIA \\ CALIFORNIA INSTITUTE OF TECHNOLOGY \\ UNIVERSITY OF CALIFORNIA \\ MONTANA STATE UNIVERSITY \\ UNIVERSITY OF NEVADA \\ NEW MEXICO STATE UNIVERSITY \\ OREGON STATE UNIVERSITY \\ UNIVERSITY OF OREGON \\ OSAKA UNIVERSITY \\ UNIVERSITY OF SOUTHERN CALIFORNIA
}

STANFORD UNIVERSITY

UNIVERSITY OF TOKYO

UNIVERSITY OF UTAH

WASHINGTON STATE UNIVERSITY

UNIVERSITY OF WASHINGTON

AMERICAN MATHEMATICAL SOCIETY
CHEVRON RESEARCH CORPORATION
TRW SYSTEMS

AMERICAN MATHEMATICAL SOCIETY

TRW SYSTEMS

NAVAL WEAPONS CENTER

Mathematical papers intended for publication in the Pacific Journal of Mathematics should be in typed form or offset-reproduced, double spaced with large margins. Underline Greek letters in red, German in green, and script in blue. The first paragraph or two must be capable of being used separately as a synopsis of the entire paper. It should not contain references to the bibliography. Manuscripts, in duplicate if possible, may be sent to any one of the four editors. All other communications to the editors should be addressed to the managing editor, Richard Arens, University of California, Los Angeles, California 90024.

Each author of each article receives 50 reprints free of charge; additional copies may be obtained at cost in multiples of 50 .

The Pacific Journal of Mathematics is published monthly. Effective with Volume 16 the price per volume (3 numbers) is $\$ 8.00$; single issues, $\$ 3.00$. Special price for current issues to individual faculty members of supporting institutions and to individual members of the American Mathematical Society: $\$ 4.00$ per volume; single issues $\$ 1.50$. Back numbers are available.

Subscriptions, orders for back numbers, and changes of address should be sent to Pacific Journal of Mathematics, 103 Highland Boulevard, Berkeley 8, California.

Printed at Kokusai Bunken Insatsusha (International Academic Printing Co., Ltd.), 7-17, Fujimi 2-chome, Chiyoda-ku, Tokyo, Japan.

PUBLISHED BY PACIFIC JOURNAL OF MATHEMATICS, A NON-PROFIT CORPORATION

The Supporting Institutions listed above contribute to the cost of publication of this Journal, but they are not owners of publishers and have no responsibility for its content or policies. 


\section{Pacific Journal of Mathematics \\ Vol. 27, No. 3}

March, 1968

Charles A. Akemann, Invariant subspaces of $C(G) \ldots \ldots \ldots \ldots \ldots \ldots . \ldots 41$

Dan Amir and Zvi Ziegler, Generalized convexity cones and their duals ... . 425

Raymond Balbes, On ( $J, M, \mathrm{~m})$-extensions of order sums of distributive

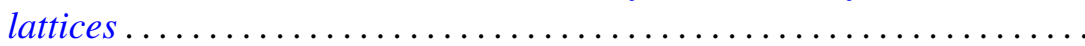

Jan-Erik Björk, Extensions of the maximal ideal space of a function algebra ........................................ 453

Frank Castagna, Sums of automorphisms of a primary abelian group ...... 463

Theodore Seio Chihara, On determinate Hamburger moment problems ..... .

Zeev Ditzian, Convolution transforms whose inversion function has complex

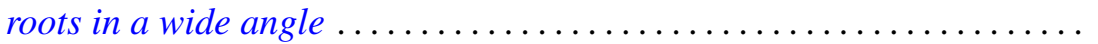

Myron Goldstein, On a paper of Rao .

Velmer B. Headley and Charles Andrew Swanson, Oscillation criteria for

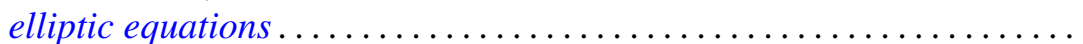

John Willard Heidel, Qualitative behavior of solutions of a third order nonlinear differential equation............................

Alan Carleton Hindmarsh, Pick's conditions and analyticity.............

Bruce Ansgar Jensen and Donald Wright Miller, Commutative semigroups

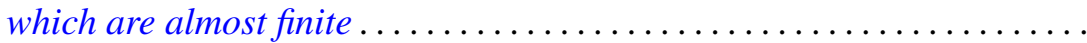

Lynn Clifford Kurtz and Don Harrell Tucker, An extended form of the

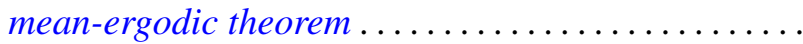

S. P. Lloyd, Feller boundary induced by a transition operator ...

Henry B. Mann, Josephine Mitchell and Lowell Schoenfeld, A new proof of the maximum principle for doubly-harmonic functions ...

Robert Einsohn Mosher, The product formula for the third obstruction ..

Sam Bernard Nadler, Jr., Sequences of contractions and fixed points ...

Eric Albert Nordgren, Invariant subspaces of a direct sum of weighted shifts...

Fred Richman, Thin abelian p-groups ...

Jordan Tobias Rosenbaum, Simultaneous interpolation in $\mathrm{H}_{2}$. II ...

Charles Thomas Scarborough, Minimal Urysohn spaces .

Malcolm Jay Sherman, Disjoint invariant subspaces..... .

Joel John Westman, Harmonic analysis on groupoids....

621

William Jennings Wickless, Quasi-isomorphism and TFM

Minoru Hasegawa, Correction to "On the convergence of resolvents of operators" 